\title{
Extraction and Quantification of Total Polyphenol Content in Different Parts of Selected Tea Cultivars
}

\author{
Simon Oduor Ochanda1, Abdul Kiptoo Faraj', John Kanyiri Wanyoko', \\ Christine Akoth Onyango ${ }^{3}$, Henrik Kipngeno Ruto ${ }^{1}$ \\ ${ }^{1}$ Kenya Agricultural and Livestock Research Organization-Tea Research Institute KALRO (TRI), Kericho, Kenya \\ ${ }^{2}$ Egerton University, Njoro, Kenya \\ ${ }^{3}$ Taita Taveta University College, Voi, Kenya \\ Email: rutohenrik@yahoo.com
}

Received 23 April 2015; accepted 26 June 2015; published 29 June 2015

Copyright @ 2015 by authors and Scientific Research Publishing Inc.

This work is licensed under the Creative Commons Attribution International License (CC BY).

http://creativecommons.org/licenses/by/4.0/

(c) (i) Open Access

\section{Abstract}

Tea (Cammelia sinensis) is the most widely consumed beverage in the world and has been reported to have unlimited health benefits due to its antioxidant properties. There is a high correlation between polyphenol compounds with antioxidant properties. Tea leaves are a major source of polyphenols. The aim of the present investigation was to determine the approximate level of polyphenols in different other parts of the tea plant to give comparative data on obtaining extracts that can be used to design products through value addition to assist in the prevention of diseases associated with oxidative stress. Twenty-one selected region specific tea varieties were used to obtain roots, flowers, leaves and barks. Leaves were prepared by microwaving and the remaining portions processed as green non-aerated teas and black aerated tea. Roots, barks, flowers were sun-dried and milled. Total polyphenol content was determined by calorimetric method using Folin-Ciocalteu reagent. The obtained results suggest that different parts of tea plant have varying numbers of total polyphenols with microwaved leaves having mean levels at $23.1 \%$, steamed leaves (non-aerated green tea) at $22.37 \%$, aerated leaves at $15.51 \%$, barks at $14.92 \%$, flowers at $10.62 \%$ and roots at $1.48 \%$.

\section{Keywords}

Tea, Polyphenols, Antioxidant Capacity, Oxidative Stress, Extract 


\section{Introduction}

Tea (Cammelia sinensis) is a widely consumed beverage [1] [2]. The tea leaves are rich in polyphenolic compounds with antioxidant properties. These polyphenols have been taunted to be of great benefits to human health and may prevent diseases associated with oxidative stress like cancer [3] [4].

Research in Kenya has contributed to discovery of new tea varieties and good agronomic practices which have led to an increase in tea yield. Tea is widely processed as black Crush Curl Tear (CTC) and 95\% of this tea is exported while the remaining tea is consumed locally. The increased production of this kind of tea has led to a significant drop in the market prices. In recent years value addition of teas has taken center stage in debates among the tea stakeholders. Value addition has come out and is seen as a means through which alternative products can be developed so as to stem out the fluctuating black CTC prices. Several value addition options are available [5] [6]. This research focuses on obtaining tea extracts with respect to tea pruning, uprooted plants and unused tea flowers [7]. The tea plant is usually pruned after some time to rejuvenate and grow rapidly, while maintaining a plucking table of about one meter. The life span of tea is usually 70 years after which tea can be uprooted [8]. At this point the tea is referred to as moribund and the production is extremely low. The teas are usually uprooted and replanting done. These activities i.e. pruning and replanting usually produce a large biomass which can be utilized by obtaining extracts for use in pharmaceutical industry and for other tea value addition chains. Conventionally pruned tea is left on the farm to rot and become manure while uprooted tea plants are used as firewood. Tea plants also have the ability to produce flowers and seeds depending on seasons and stress levels. Phyto-chemicals use is not only limited to foods but also used to manufacture drugs [9] [10]. Residues from the extraction can be utilized as manure and animal feeds accordingly [5]. Ascertaining the polyphenol levels of the different tea parts will enable researchers to appropriately utilize the tea plant in relevant areas of value addition.

\section{Materials and Methods}

\subsection{Materials}

Twenty one region specific tea varieties were collected to represent different tea growing regions. The tea varieties were acquired from Kenya Agricultural and Livestock Research Organization-Tea Research Institute(KALRO-TRI).

\subsection{Sample Preparation}

Four different parts of the tea plant were used including; leaves, barks, flowers and roots. Barks from the clonal samples were obtained from the field (Table 1). Flower samples were harvested and sundried and milled while bark and root samples were washed to remove soil particles, sun dried and milled in to fine powder. Leaves were divided into three portions, portion 1 was micro waved and milled while the portion 2 and portion 3 were processed into black (aerated) and green (non-aerated) tea respectively.

\subsection{Total Polyphenol Assay}

Extraction of catechins and total polyphenols were done according to the procedure by ref [11]. Ground tea samples $(0.2 \mathrm{~g})$ were weighed into graduated extraction tubes and $5 \mathrm{ml}$ of $70 \%$ hot methanol/waterv $/ \mathrm{v}(\mathrm{MeOH})$ added, stoppered and mixed under vortex. Incubation followed at $70^{\circ} \mathrm{C}$ for $10 \mathrm{~min}$ (water bath) with vortexing at 0,5 and $10 \mathrm{~min}$, cooling to rtp and then centrifuging (3000 rpm) for $10 \mathrm{~min}$. A second extraction was performed and the extracts combined and made up to $10 \mathrm{ml}$ with cold methanol/water v/v (MeOH) at 70\%. Extracted samples were complexed using 75\% sodium carbonate and 10\% Folin-ciocalteu reagent (phenol) and absorbance read at $765 \mathrm{~nm}$. The total polyphenol content was expressed as a percentage by mass on a sample dry matter [11] [12].

\section{Results and Discussion}

Total polyphenol content obtained for the different parts of tea plant in 21 selected clones were represented in graphs (Figures 1-4). The results compared polyphenols levels in clones, different parts of the tea plant and different leaf processing methods. Values are means of three replicates. 


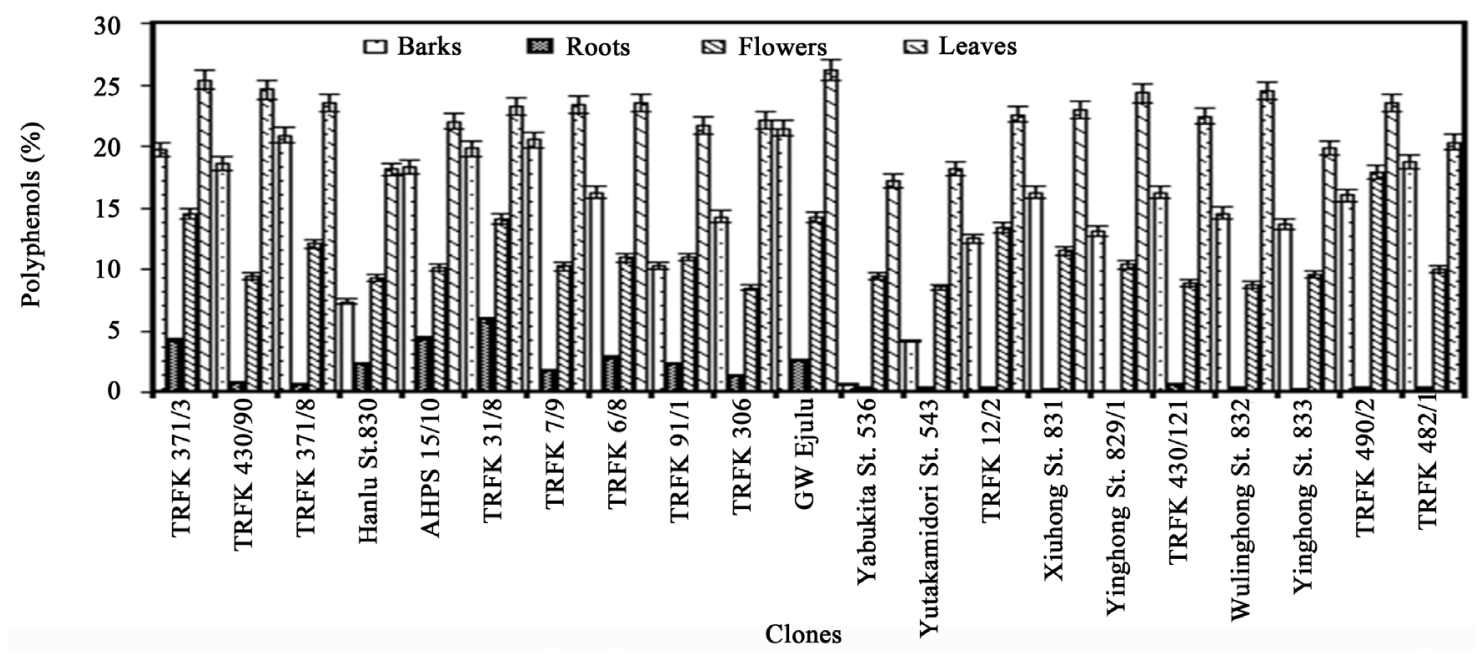

Figure 1. Total polyphenol content in barks, roots, flowers and green tea leaves in 21 selected tea varieties.

Table 1. Table showing tea varieties used to acquire different parts of the tea plant.

\begin{tabular}{|c|c|c|}
\hline S/No. & Tea clone & Origin \\
\hline 1. & TRFK 371/3 & TRFK Kenya \\
\hline 2. & TRFK 430/90 & TRFK Kenya \\
\hline 3. & TRFK 371/8 & TRFK Kenya \\
\hline 4. & Hanlu St. 830 & China \\
\hline 5. & AHP S15/10 & JFK, Kenya \\
\hline 6. & TRFK 31/8 & TRFK Kenya \\
\hline 7. & TRFK 7/9 & TRFK Kenya \\
\hline 8. & TRFK 6/8 & TRFK Kenya \\
\hline 9. & TRFK 91/1 & TRFK Kenya \\
\hline 10. & TRFK 306 & TRFK Kenya \\
\hline 11. & GW Ejulu & George Williamson, Kenya \\
\hline 12. & Yabukita St. 536 & TRFK Kenya \\
\hline 13. & Yutakamidori St. 543 & TRFK Kenya \\
\hline 14. & TRFK $12 / 2$ & TRFK Kenya \\
\hline 15. & Xiuhong St. 831 & China \\
\hline 16. & Yinghong St. 829/1 & China \\
\hline 17. & TRFK 430/121 & TRFK Kenya \\
\hline 18. & Wulinghong St. 832 & China \\
\hline 19. & Yinghong St. 833 & China \\
\hline 20. & TRFK 490/2 & TRFK Kenya \\
\hline 21. & TRFK 482/1 & TRFK Kenya \\
\hline
\end{tabular}




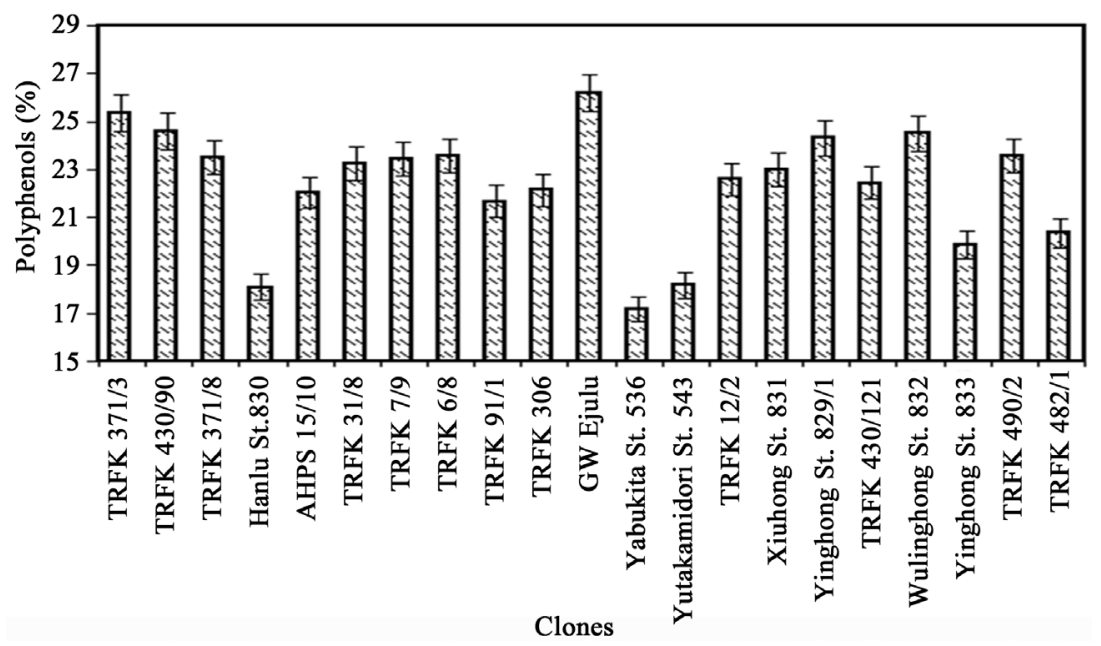

Figure 2. Total polyphenol content of non-aerated tea leaves of 21 selected tea cultivars.

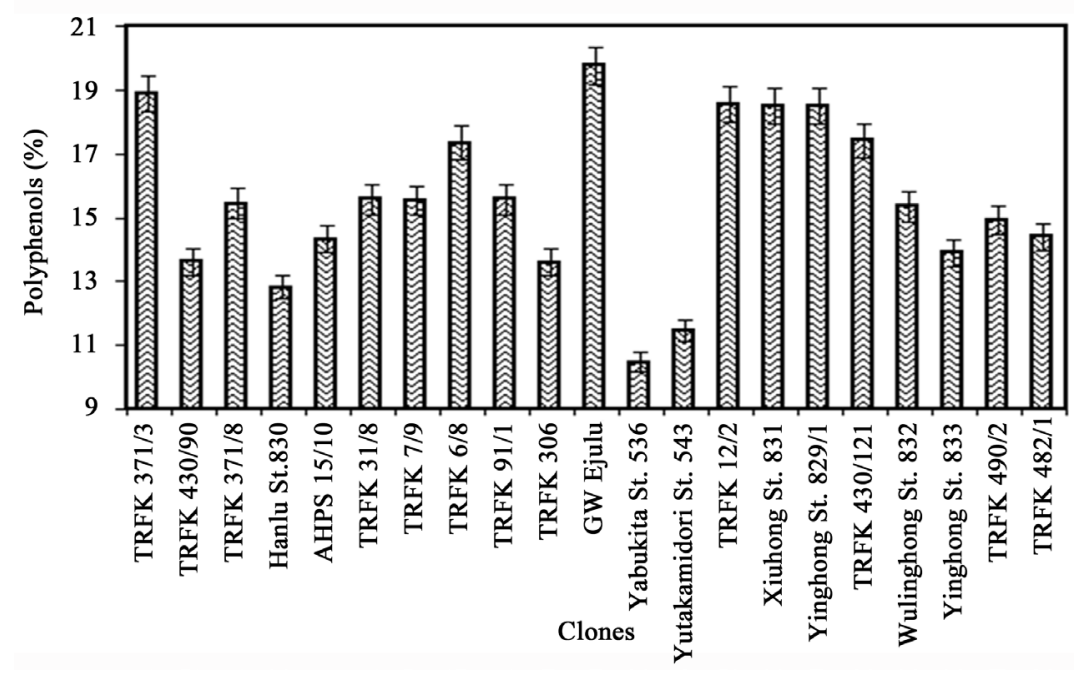

Figure 3. Total polyphenol content of aerated (black) tea leaves of 21 selected tea cultivars.

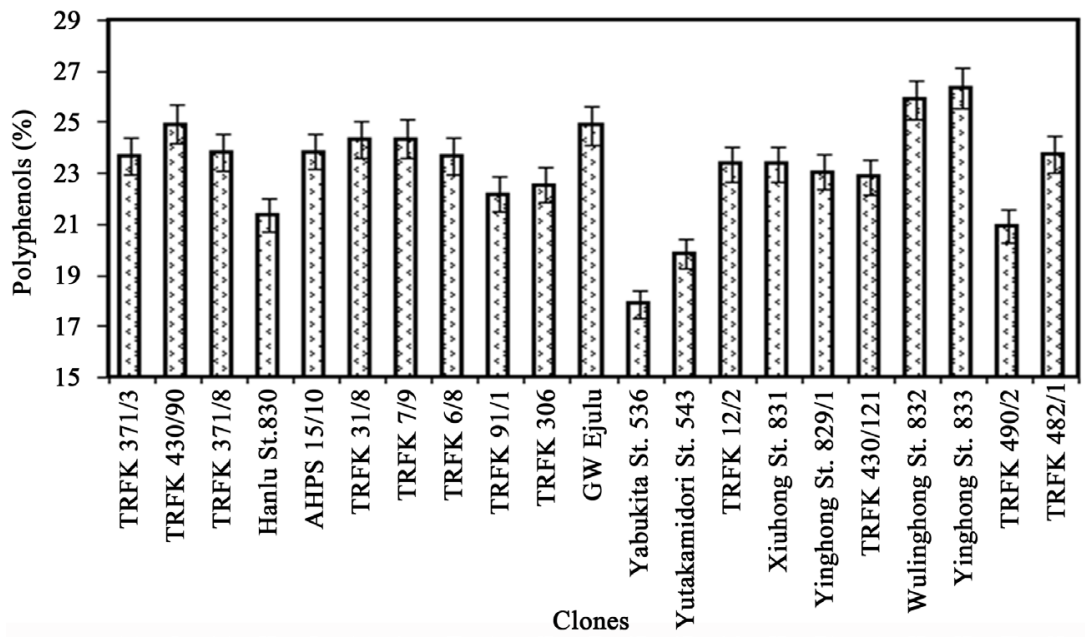

Figure 4. Total polyphenol content of micro-waved tea leaves of 21 selected tea cultivars. 
The bark of GW Ejulu had the highest levels of polyphenols at 21.44\% with TRFK 371/8 at 20.91\% while Yabukita St. 536 had the least levels at $0.63 \%$ of polyphenols. The roots of clone TRFK 31/8 had the highest level of total polyphenols at 5.36\% while clone Yinghong St. 833 had the least at $0.1 \%$. Flowers clone TRFK 490/2 had the highest levels of total polyphenols at $17.87 \%$ while those of TRFK 306 had the lowest polyphenol content at $8.51 \%$.

Non-aerated tea leaves exhibited relatively higher content of total polyphenols compared to the other parts of the plant in which clone GW Ejulu had the highest levels at 26.2\% whereas clone Yabukita St. 536 had the least at $17.2 \%$ (Figure 4). Aerated leaves showed a decline in polyphenol content as compared to the non-aerated counter parts. During aeration polyphenol oxidase converts catechins mainly present in un-aerated tea to thearubigins and theaflavins [11] [13]. This explains the reduction in total polyphenol content. These results were in agreement with work done by ref [14] in Argentina also showed that processing technologies have an effect on polyphenol levels in tea.

The mean total polyphenol content was significantly different at $P<0.05$, between plant parts and processing method. Leaves had the highest levels at $23.17 \%$ for the micro-waved tea, $22.37 \%$ for un-aerated (green) tea and $15.51 \%$ for aerated (black) leaves. Other parts of the plant had levels of $14.92 \%$ for barks, $10.62 \%$ for flowers and $1.48 \%$ for roots.

\section{Conclusion}

This study has demonstrated that polyphenols are present in all the investigated tea parts with the leaf having the highest amounts. Barks of GW Ejulu variety had levels of up to $21 \%$ which are comparable to the mean levels in leaves. These results provide a strong insight and form a strong basis for value addition in the tea industry. It can be recommended that extracts from tea parts can be obtained when tea is pruned or uprooted. Optimization of leaf processing techniques for beverage use should also be optimized for consumers to obtain optimum amount of these vital phytochemicals which correlate to high antioxidant capacity and increased health promoting properties.

\section{Acknowledgements}

The authors would like to acknowledge National Commission for Science, Technology and Innovation (NACOSTI) and Kenya Agricultural Research Organization-Tea Research Institute (KALRO-TRI) for their financial support.

\section{References}

[1] Ferruzzi, M.G. (2010) The Influence of Beverage Composition on Delivery of Phenolic Compounds from Coffee and Tea. Physiology \& Behavior, 100, 33-41. http://dx.doi.org/10.1016/j.physbeh.2010.01.035

[2] Sumpio, B.E., Cordova, A.C., Berke-Schlessel, D.W., Qin, F. and Chen, Q.H. (2006) Green Tea, the 'Asian Paradox', and Cardiovascular Disease. Journal of the American College of Surgeons, 202, 813-825. http://dx.doi.org/10.1016/j.jamcollsurg.2006.01.018

[3] Da Silva Pinto, M. (2013) Tea: A New Perspective on Health Benefits. Food Research International, 53, 558-567. http://dx.doi.org/10.1016/j.jamcollsurg.2006.01.018

[4] Ochanda, S.O., Wanyoko, J.K. and Ruto, H.K. (2015) Effect of Spices on Consumer Acceptability of Purple Tea (Camellia sinensis). Food and Nutrition Sciences, 6, 703-711. http://dx.doi.org/10.4236/fns.2015.68073

[5] Njuguna, D., Wanyoko, J., Kinyanjui, T. and Wachira, F. (2013) Polyphenols and Free Radical Scavenging Properties of Kenyan Tea Seed Oil Cake. International Journal of Research in Chemistry and Environment, 3, 86-92.

[6] Moseti, K.O., Kinyanjui, T., Wanyoko, J.K., Kurgat, J.K., Too, J.C., Omondi, K.G. and Wachira, F.N. (2013) Fe, Zn, $\mathrm{Cu}, \mathrm{Pb}$ and $\mathrm{Cd}$ in Tea Grown and Marketed in Kenya; A Quantitative Assessment. International Journal of Environmental Protection, 3, 24-30.

[7] Ochanda, S.O., Wanyoko, J.K., Onyango, C.A., Faraj, A.K. and Kamunya, S.M. (2012) Screening of Suitable Clones for Un-Aerated Tea Production. African Journal of Horticultural Science, 6, 118-135.

[8] Magambo, M.J.S. and Cannell, M.G.R. (1981) Dry Matter Production and Partition in Relation to Yield of Tea. Experimental Agriculture, 17, 33-38. http://dx.doi.org/10.1017/S0014479700011200

[9] Owuor, P.O. and Obanda, M. (2007) The Use of Green Tea (Camellia sinensis) Leaf Flavan-3-ol Composition in Predicting Plain Black Tea Quality Potential. Food Chemistry, 100, 873-884.

http://dx.doi.org/10.1016/j.foodchem.2005.10.030 
[10] Schneider, C. and Segre, T. (2009) Green Tea: Potential Health Benefits. American Family Physician, 79, 591-594.

[11] Karori, S.M., Wachira, F.N., Wanyoko, J.K. and Ngure, R.M. (2007) Antioxidant Capacity of Different Types of Tea Products. African Journal of Biotechnology, 6, 2287-2296.

[12] Lelgo, S.K., Kamunya, S.M., Ochanda, S.O. and Wanyoko, J.K. (2011) The Effect of Processing Technique on Biochemical Composition of Adapted Kenyan and Introduced Japanese and Chinese Tea Germplasm. Tea, 32, 22-23.

[13] Kerio, L.C., Wachira, F.N., Wanyoko, J.K. and Rotich, M.K. (2012) Characterization of Anthocyanins in Kenyan Teas: Extraction and Identification. Food Chemistry, 131, 31-38. http://dx.doi.org/10.1016/j.foodchem.2011.08.005

[14] Anesini, C., Ferraro, G.E. and Filip, R. (2008) Total Polyphenol Content and Antioxidant Capacity of Commercially Available Tea (Camellia sinensis) in Argentina. Journal of Agricultural and Food Chemistry, 56, 9225-9229. http://dx.doi.org/10.1021/jf8022782 